\title{
Measures of explicit and implicit in motor learning: what we know and what we don't
}

\author{
Jana Marescha, Liad Mudrikb, Opher Donchin ${ }_{c}$
}

a Department of Brain and Cognitive Sciences, Ben Gurion University of the Negev, Be'er Sheva, Israel

¿ Sagol School of Neuroscience and School of Psychological Sciences, Tel Aviv University, Tel Aviv, Israel. Email address: mudrikli@,tamex.tau.ac.il.PO Box 39040,Tel Aviv69978, Israel

. Department of Biomedical Engineering and Zlotowski Center for Neuroscience, Ben-Gurion University of the Negev, Be'er Sheva, Israel.Email address:_doncbin@,bgu.ac.il.P.O.B.653, Beer-Sheva 8410501, Israel

Corresponding author:

Jana Maresch

Motor Learning Lab, Ben Gurion University of the Negev

Marcus Family Campus

Ben Gurion Drive 1

8410501 Be'er Sheva, Israel

+974-5566 49697

jana.maresch@gmail.com 
Multiple different processes are known to contribute to sensorimotor learning, and adaptation tasks have been a key tool in characterizing these underlying processes. Recently, much interest has focused on quantifying the explicit and implicit components of motor adaptation using a variety of methods. The methods differ in their underlying assumptions and ideas. In some cases, they yield similar findings, in others they do not. We review the literature with a focus on the agreement and inconsistencies between different measures of explicit adaptation. Some aspects of explicit adaptation seem robust across different measurements: the fast time constant of the explicit system and the slow time constant of the implicit system, for instance. Other aspects seem to reflect quite differently across measures: for example, the extent to which explicit and implicit combine linearly. To help understand these differences, we explored ideas of explicit and implicit learning in the context of the larger field of cognitive science. We found that non-linearity and a possible bias in the measurements make explicit and implicit learning difficult to measure across different fields within cognitive science. We relate this back to the study of motor adaptation, arguing that the only way forward is through a strong experimental characterization of the phenomenology of our visuomotor adaptation and a rich set of models to test on it. 
Intuitively, it seems we are aware of our motor behavior, but we don't really understand what that means or what difference it makes. Indeed, awareness of our motor behavior may mean different things. In some cases, we are more aware of our motor plans than our motor behavior: while walking through a busy street, we may be more aware of planning our path forward than we are aware of our balance or our foot placement or the coordination of muscle activity and joint movements that supports either of these (Bernstein 1967). In other situations, the opposite can happen: while walking over a wooden log, we typically bring our attention to balance and foot placement while the path forward may fade into the background. As a further illustration, we now ask the reader to momentarily bring their attention to their own breathing. See? Suddenly, motor behavior that was completely outside the scope of awareness becomes a part of experience. This phenomenon is well recognized and widely discussed in the sensory domain. One well-known example: suddenly becoming aware of the feeling of a shirt on our neck or noticing a background noise that was previously outside awareness (Block 1995). Thus, awareness of motor behavior is flexible, much like awareness of external stimuli in the environment. Thus, it makes sense to place the study of awareness in motor behavior within the context of the larger study of the functions of consciousness and the scope of unconscious processing. Indeed, it may even be hoped that findings in the motor system will shed light on some of the difficult questions in the study of consciousness whose answers have long been sought (for reviews of ongoing debates, see Hassin (2013), Kouider and Dehaene (2007), Mudrik, Faivre et al. (2014), van Gaal, De Lange et al. (2012)). (For now, we will use aware, explicit and conscious interchangeably but the terms differ and we provide clear definitions for them below.)

An intriguing case study of explicit processes in motor behavior is that of motor learning. Different aspects of motor learning may require more or less explicit processes, and the ratio between explicit and implicit ones may change through the process of learning. Learning to make a basket in basketball involves explicitly learning about hand placement and stance and implicit development of coordination of leg and arm movements. A basketball coach may explicitly bring some aspect of the behavior, such as follow through, to the players' attention in order to help them improve. After practice, this aspect may eventually become automatic and no longer require attention. Learning to ride a bicycle is classically considered an example of procedural, implicit learning (Koziol and Budding 2012) although our parents typically try to explicitly explain how to pedal and maintain balance, while running behind their kids.

The involvement of explicit and implicit processes in motor learning (and in motor behavior in general) has been investigated in many forms and in different fields. Both in sports sciences and in 
rehabilitation sciences, fundamental research asks how to enhance motor skill learning through explicit instruction (for review see: Kal, Prosée et al. 2018). Other fields - cognitive psychology, philosophy and motor systems - focus on dissociating explicit and implicit processes with the goal of characterizing and quantifying the contribution of each.

Yet this is not an easy feat; when inspecting the literature, even when focusing only on motor learning, we see that the main constructs- awareness/consciousness, attention, explicit vs. implicit - are often not properly defined. Accordingly, they are probed using a variety of paradigms and methods that often reflect conflicting underlying assumptions. This often leads to some confusion in interpreting their results and formulating a comprehensive theory of the roles of explicit and implicit processes in motor learning. Here, we aim to mitigate some of this confusion by summarizing the different ideas about explicit and implicit learning used in the motor field and the different methods to measure them. We will then place these in the context of the larger field of cognitive science and draw insights from how the implicit/explicit distinction has been used to describe other phenomena. Ultimately, we will reapply these insights to the study of motor control.

\section{Terminology}

Our first aim, accordingly, is to clarify the critical terms we use. The reader might have been troubled by a cavalier attitude with different terms used interchangeably. This reflects a broader problem in the literature, where the terms referring to conscious, unconscious, aware, unaware, explicit, implicit, declarative and procedural are used both interchangeably and inconsistently. This renders comparisons between different approaches and fields difficult. Let us try to be more careful. We will define the different terms and specify how they will be used throughout this review.

In the fields of cognitive science, it has been useful to distinguish between one's awareness of a sensory or a perceptual event (being aware of, or having access to, a picture of a face that was flashed on screen) and one's awareness of internal cognitive or affective or evaluative processes (being aware of the feelings evoked by that face). Thus, conscious/unconscious and aware/unaware are often used as synonymous pairings to indicate situations when subjects perceive or do not perceive a perceptual event (e.g., Dehaene, Changeux et al. (2006), Lamme (2006)). While the event is typically triggered by an external stimulus, one can also speak of being aware of a sensory event in one's dream or imagination. Explicit/implicit, on the other hand, are often used to describe situations where the stimulus is consciously perceived, and the subject does or does not have access to the way the stimulus affects their behavior (Reber 1989, Cleeremans, Destrebecqz et al. 1998). This definition has been 
Table 1 Differences between procedural/declarative and implicit/explicit knowledge of a task.

\begin{tabular}{|c|c|c|c|c|c|}
\hline & & $\begin{array}{l}\text { Ability to perform but } \\
\text { not describe }\end{array}$ & $\begin{array}{l}\text { Ability to describe } \\
\text { but not perform }\end{array}$ & $\begin{array}{l}\text { Ability to perform } \\
\text { and to describe }\end{array}$ & $\begin{array}{c}\text { Neither ability to perform } \\
\text { nor to describe }\end{array}$ \\
\hline & & $\begin{array}{l}\text { Procedural but not } \\
\text { declarative }\end{array}$ & $\begin{array}{l}\text { Declarative but not } \\
\text { procedural }\end{array}$ & $\begin{array}{l}\text { Procedural and } \\
\text { declarative }\end{array}$ & $\begin{array}{c}\text { Neither procedural or } \\
\text { declarative }\end{array}$ \\
\hline $\begin{array}{l}\text { Knowledge that I } \\
\text { can't access by } \\
\text { choice }\end{array}$ & Implicit & $\begin{array}{c}\text { Balancing on a bicycle } \\
\text { but not knowing how to } \\
\text { describe it }\end{array}$ & $\begin{array}{l}\text { Knowing how to } \\
\text { describe bicycle } \\
\text { balance but not being } \\
\text { able to do it }\end{array}$ & $\begin{array}{c}\text { Balancing on a bicycle } \\
\text { and knowing how to } \\
\text { describe it }\end{array}$ & Impossible by definition \\
\hline $\begin{array}{c}\text { An internal process I } \\
\text { can report }\end{array}$ & Explicit reportable & $\begin{array}{l}\text { Being able to say that } \\
\text { I'm pedaling but not } \\
\text { being able to describe } \\
\text { it }\end{array}$ & $\begin{array}{c}\text { Describing pedaling a } \\
\text { bike but not being able } \\
\text { to tell if I'm pedaling or } \\
\text { not }\end{array}$ & $\begin{array}{c}\text { Describing pedaling a } \\
\text { bike, and being able to } \\
\text { tell if I'm pedaling or } \\
\text { not }\end{array}$ & Impossible by definition \\
\hline $\begin{array}{l}\text { An internal process I } \\
\text { can control }\end{array}$ & Explicit controllable & $\begin{array}{l}\text { Being able to pedal by } \\
\text { choice, but not being } \\
\text { able to describe } \\
\text { pedaling }\end{array}$ & Impossible by definition & $\begin{array}{l}\text { Being able to pedal by } \\
\text { choice, and being able } \\
\text { to describe it }\end{array}$ & Impossible by definition \\
\hline
\end{tabular}

applied in a variety of fields within the cognitive sciences (implicit/explicit attitudes, Perugini (2005), Nosek (2007), implicit/explicit learning, Chun and Jiang (1998), DeKeyser (2008); and implicit/explicit memory Graf and Schacter (1985)). Notably, researchers do sometimes use the terms 'conscious/unconscious' to refer to internal processes, since subjects are indeed not consciously aware of their implicit processes (Rothkirch and Hesselmann 2017). Yet the opposite is not true: the terms 'explicit/implicit' are not used, to our knowledge, to refer to consciously perceived and consciously unperceived stimuli. In this review, we will adopt the distinction so that the terms are dissociated: 'conscious/unconscious' or 'aware/unaware' refers to having access to perceptual events, while 'implicit/explicit' refers to having access to some internal process evoked by/performed on that event. Applying these ideas to the study of motor control leads us to extend the terms 'aware' and 'unaware' to the experience of one's own motor behavior and to reserve the terms 'implicit' and 'explicit' for the underlying internal processes. To make this concrete, imagine again walking over a wooden log and apply the different terms carefully. We are aware of our careful foot placement; we might use an explicit strategy to maintain balance, such as extending our arms as if walking on a tightrope; our postural corrections will be both implicit and unaware, we will not experience them nor will we experience choosing them. 
One of the central ideas in the current review is that explicit access to motor processes is complex, possibly involving different sorts of access to multiple underlying processes. To advance a specific complexity, we distinguish an ability to describe the strategy I'm currently applying from an ability to deliberately choose to implement it or stop using it. We call these reportable explicit knowledge and controllable explicit knowledge. To make this concrete, let's look at bicycle riding. Balancing on the bike happens at an implicit level: I can neither choose to balance nor describe how I do it. Pedaling the bike is explicit in both senses: I can say when I'm doing it and I can choose to start and stop. Turning the bike is explicitly controlled although many aspects of it are not accessible to report: I decide to turn, and I may report that I turn the handlebars, but many aspects of turning such as the appropriate lean and speed adjustments are not easily reported. On the other hand, when the bike hits a bumpy part of the road, I may be able to report my strategy - I slow down, grip the handlebars more tightly, and come up off my seat - but most of the response happens without being under my explicit control.

To our view, all of these distinctions are separate from the distinction between declarative and procedural knowledge, which has served an important role in our understanding of memory. The procedural / declarative distinction is often conflated with the explicit / implicit distinction, both outside the motor field (Dharani 2014) and within it (Huberdeau, Krakauer et al. 2019, Hadjiosif and Krakauer 2020). Procedural knowledge is knowledge that can be performed, while declarative knowledge is knowledge that can be described. In table 1, we elaborate on the example of bicycle riding and dissociate implicit, explicit reportable, and explicit controllable processes from those about which we have procedural and declarative knowledge. As the table makes clear, we can have declarative knowledge of implicit processes and procedural knowledge of processes that are explicitly accessible. In sum, we refer to subjects as aware when they have access to (they experience) a perceptual stimulus, and internal event, or changes in their own behavior, we say they are processing explicitly when subjects are able to report on or have control of the internal processes taking place, we say they have declarative knowledge when they can describe the world or internal processes, even if they have not experienced them. The distinction between procedural and declarative knowledge is a rich one and its application to motor behavior will undoubtedly be fruitful. However, there is no real work on this yet. For the remainder of the review we focus on the distinction between explicit and implicit processes.

\section{Adaptation - a simplifying tool to study processes in motor learning}

Different paradigms have been employed to study explicit and implicit processes in motor learning.

One approach is to use a simplified task, which allows the researchers to observe learning while 
manipulating different kinds of inputs and behaviors. By reducing the amount of external and internal factors that may influence learning, the researchers can disentangle explicit from implicit processes and consider possible influencing factors.

Motor adaptation provides a good opportunity for this approach: it is a simple form of motor learning where recent interest has focused on explicit and implicit processes. Unlike acquiring a new skill, adaptation does not require acquisition of a new pattern of muscle activity, but rather of a new mapping between well-known movements and a spatial goal (Krakauer and Mazzoni 2011). A common experimental approach to studying motor adaptation is to impose a systematic perturbation while subjects perform simple reaching movements. For example, a force field may be applied to the hand (Shadmehr and Mussa-Ivaldi 1994) or visual feedback may be altered by inverting prisms (Welch 1986) or visuomotor rotations (Krakauer, Pine et al. 2000). The latter is a task in which a cursor representing the hand is rotated clockwise or counterclockwise relative to its origin, resulting in a mismatch between the hand and the cursor movement. Participants only see the motion of the cursor and slowly adapt to the visuomotor distortion. In recent years, research has focused on the constituent components of visuomotor adaptation, and methods have been developed to separate explicit and implicit components.

\section{Explicit and implicit processes in visuomotor rotation tasks}

After reviewing the literature on explicit and/or implicit processes during adaptation, we identified 32 articles that explicitly deal with quantifying these processes under different conditions. We found seven major methodologies for quantifying the processes used by the different researchers. Table 2 provides an overview of these methodologies. The key point of the table is that the different methods can be clearly divided according to three different underlying assumptions (yellow shaded columns): the first states that a process is explicit if a subject can choose to turn it on or off (i.e., control it). The second assumption holds that a process is explicit if the subject can accurately report on it. And the third assumption (which, as the table shows, is less common) is that explicit processes require cognitive resources.

Aside from classifying the different methods according to assumptions, they differ in additional ways. To give a flavor of these differences, we identified several common features that delineate the different methods (blue shaded columns). For instance, some studies use trial-by-trial measurements and others rely on blocked designs. The former allows the researchers to continuously track explicit, implicit or 
Table 2 The seven major methodologies used in order to measure explicit and/or implicit processes during adaptation to visuomotor rotations.

\begin{tabular}{|l|c|c|c|c|c|c|c|c|}
\hline & \multicolumn{3}{|c|}{ Assumptions } & \multicolumn{3}{c|}{ Task features } & \multicolumn{2}{c|}{$\begin{array}{c}\text { Directly measured } \\
\text { process }\end{array}$} \\
\hline & $\begin{array}{c}\text { Explicitcan } \\
\text { be } \\
\text { controlled }\end{array}$ & $\begin{array}{c}\text { Explicitis is } \\
\text { reportable }\end{array}$ & $\begin{array}{c}\text { Explicit } \\
\text { requires } \\
\text { cognitive } \\
\text { resources }\end{array}$ & $\begin{array}{c}\text { Memory } \\
\text { based }\end{array}$ & $\begin{array}{c}\text { Trial-by-trial } \\
\text { measureme } \\
\text { nt }\end{array}$ & Blocked & Explicit & Implicit \\
\hline Aftereffect & $\checkmark$ & & & & & $\checkmark$ & & $\checkmark$ \\
\hline PDP & $\checkmark$ & & & $\checkmark$ & & $\checkmark$ & & $\checkmark$ \\
\hline $\begin{array}{l}\text { Clamped visual } \\
\text { feedback }\end{array}$ & $\checkmark$ & & & & $\checkmark$ & & & $\checkmark$ \\
\hline Verbal report & & $\checkmark$ & & & $\checkmark$ & $\checkmark$ & $\checkmark$ & \\
\hline $\begin{array}{l}\text { Pointing with left } \\
\text { hand }\end{array}$ & & $\checkmark$ & & & $\checkmark$ & $\checkmark$ & $\checkmark$ & \\
\hline Explicit posttest & & $\checkmark$ & & $\checkmark$ & & $\checkmark$ & $\checkmark$ & \\
\hline $\begin{array}{l}\text { Restriction of prep } \\
\text { time }\end{array}$ & & & $\checkmark$ & & $\checkmark$ & & & $\checkmark$ \\
\hline
\end{tabular}

both processes, allowing the researchers to obtain an estimate of the time course of the processes. It is worth noting that some trial-by-trial measures like verbal report can interfere with the adaptation

processes (Newell and Shanks 2014, Timmermans and Cleeremans 2015). As can be seen in the table, memory-based methods always use a blocked design: subjects are tested at the end of a pre-defined block on the knowledge accumulated to that point. However, blocked designs are not necessarily memory-based, as is the case for the aftereffect, which we will describe in detail later.

It is also possible to distinguish between methodological approaches by whether they measure both the implicit and explicit processes directly (green shaded columns). Sometimes, the measure of one process is derived from the direct measurement of the other. In these cases, the authors assume that the processes are additive. In the following paragraphs, we describe each of the basic methodological approaches, highlighting its underlying assumptions, its features, and what types of processes it is designed to measure.

The aftereffect, the process dissociation procedure (PDP (Jacoby 1991)) and the clamped visual feedback are all based on the assumption that explicit processes can be controlled. The aftereffect task is administered at the end of the adaptation stage. The perturbation is turned off, and subjects are asked to 'aim straight towards the target'. The remaining error they produce (held to be outside their control, as they are aiming to move straight) is considered to index the implicit adaptation that contributed to their behavior during the previous stage. The PDP uses a measure called 'exclusion'. These trials are also conducted at the end of the adaptation stage, and subjects are asked to reproduce the behavior from before any rotation was applied (Werner, van Aken et al. 2015). That is, to exclude or ignore the 
adapted behavior they developed during the first stage. And so, subjects should recall what they did at the beginning of the experiment and reproduce that behavior. Hence, this measure is memorybased. Again, the remaining error they demonstrate (i.e., the 'uncontrolled' part of their behavior) is held to index implicit adaption. Quite differently, the clamped visual feedback method relies on preventing explicit strategy from developing already at the beginning of the task (Morehead, Taylor et al. 2017). Subjects are informed that the cursor will be rotated, and are also informed of the extent of the rotation. They are then asked to ignore the error between the cursor representing their hand and the target. Despite these instructions, subjects nevertheless adapt to the rotation. This uncontrolled adapted behavior reflects implicit adaptation measured on a trial-by-trial basis. Thus, all three methods measure implicit contributions to adaptation. They also differ in important ways: clamped feedback provides a trial-by-trial estimate where the other two methods use a blocked approach, usually estimating implicit adaptation only at the end of adaptation.

In a second group of methods, verbal report (Taylor, Krakauer et al. 2014), pointing with left hand (Bond and Taylor 2017) and explicit posttest (Hegele and Heuer 2010) capture reportable explicit processes of adaptation. In verbal report, subjects are presented with an array of visual landmarks surrounding the target and are asked to report their intended aiming direction verbally before each trial, providing a trial-by-trial estimate of their explicit strategy. Then additivity is assumed in order to derive the implicit adaptation from the explicit. Pointing with left hand is similar to the verbal report, but here, subjects tap a touch screen with their left hand to indicate the intended aiming direction. Here, again, implicit adaptation is inferred using the assumption of additivity. The explicit posttest task, as the name suggests, is only performed at the end of adaptation and it is a memory-based measure. In this method, subjects are presented with a line centered at the start location. The orientation of the line is opposite to the imaginary line between the center and the visual target and subjects are asked to instruct the experimenter to rotate the line until it matches the aiming direction appropriate to reach the target. The explicit posttest has been used in combination with the aftereffect to provide direct measurements of both explicit and implicit and the end of adaptation.

Currently, only one approach has leveraged the idea that explicit adaptation requires cognitive resources and this is done using restriction of preparation time. It has been repeatedly suggested that cognitive processes, such as explicit strategizing and mental rotation, are an important part of explicit adaptation to visuomotor rotations (Eversheim and Bock 2001, Anguera, Reuter-Lorenz et al. 2010, Taylor, Krakauer et al. 2014, Bond and Taylor 2015). These processes are known to require longer 
response times (Georgopoulos and Massey 1987). Thus, it is reasonable to think that restriction of preparation times should prevent the cognitive contributions to adaptation so that only implicit adaptation processes can take place (Fernandez-Ruiz, Wong et al. 2011, Haith, Huberdeau et al. 2015, Leow, Gunn et al. 2017). This approach has been used by different authors to probe implicit processes, without trying to estimate explicit contribution to adaptation.

\section{Congruencies and incongruences between studies in visuomotor rotation tasks}

Table 3 gives an overview of articles using one or more of the methods described above. We show certain characteristics of explicit re-aiming and implicit adaptation in the top row of the table: the first three are adapted from Table 2, the last four are key characteristics of explicit and implicit processes. These characteristics are sorted from left to right: from robust results across studies to partially/mainly incongruent results between different studies. An empty cell indicates that a study did not provide information about the characteristic. An X means that the study shows the opposite of what is claimed in the column header and a checkmark indicates agreement with the claim.

The characteristic on which there is broadest agreement (fourth column) is the timescale of explicit and implicit adaptation: implicit adaptation is slow and continues rising throughout adaptation whereas explicit re-aiming has a fast initial rise and then drops off. This robust finding has parallels in other motor learning fields, such as in models of skill acquisition (Fitts and Posner 1967). In support of this finding, researchers have independently established the existence of two distinguishable time scales in motor adaptation: a fast process, which learns rapidly but has poor retention, and a slow process, which learns slowly but retains the gained information (Smith, Ghazizadeh et al. 2006).

Another result reported by a number of researchers is that implicit adaptation is independent of many details of the task. However, recent studies have revealed factors that do influence the rate of implicit adaptation. These include the size of errors, the presence of rewards, or the distribution of the targets in space (Bond and Taylor 2015, McDougle, Bond et al. 2015, Leow, Gunn et al. 2017, Kim, Parvin et al. 2019, Yin and Wei 2020). One possibility that needs further study is that all of these different manipulations are actually confounded with the rate at which subjects are rewarded. If so, it may well be true that, while implicit adaptation is independent of many task details, it can still be influenced by very specific details such as reward frequency. Further work on this issue is necessary, but it seems reasonable to expect a consensus to emerge regarding the factors that influence the rate of implicit adaptation. 
The sixth column of table 3 addresses the extent to which different measures of explicit and implicit produce consistent results. A large number of studies combine multiple measures of explicit and implicit - most often aftereffect is combined with another measure such as report or error clamp. In a few cases, results are consistent across methods (Brudner, Kethidi et al. 2016, Morehead, Taylor et al. 2017), but, as the table shows, they usually aren't. Consistency across measures can depend heavily on details of the task, even to the point of changing the direction of the difference (Bromberg, Donchin et al. 2019, Maresch, Werner et al. 2020). Aftereffect often indicates less implicit adaptation than other methods (Taylor, Krakauer et al. 2014, Leow, Gunn et al. 2017, de Brouwer, Gallivan et al. 2018, Kim, Parvin et al. 2019, Wilterson and Taylor 2019, Yin and Wei 2020). Maresch et al. 2020 compared the report measure with intermittent exclusion and the PDP. Report showed more explicit re-aiming than the estimated contribution of explicit processes as calculated using the exclusion task (the equivalent to the implicit calculated from the report). They found more consistency across measures during reporting than when subjects did not report and that the PDP showed less explicit processing than both other measures (Maresch, Werner et al. 2020).

Thus, there are characteristics of explicit and implicit adaptation that are consistent and robust across studies, and there are findings that are more controversial. We discussed the possibility that differences in task design may affect the expression of certain characteristics in the different studies and therefore lead to differences between studies. Another cause for incongruences between studies can be the two major assumptions that are commonly made in the motor field: The first is that measures of explicit and implicit are unbiased. We discuss bias in the next section. The second is that actual behavior is a simple sum of an explicit component and an implicit component. We call this second assumption linearity. The possibility was first raised for motor adaptation by Sülzenbrück and Heuer (2009), who showed that linearity held in multiple experiments. More widely known is the seminal paper of Mazzoni and Krakauer which showed that the amount of adaptation after subjects were given an explicit strategy was equal to the aftereffect plus the size of the strategic re-aiming (Krakauer and Mazzoni 2011). Linearity is a convenient assumption. First, it connects the two components of explicit and implicit adaptation to a two-state model of adaptation originally proposed to explain savings (Smith, Ghazizadeh et al. 2006). Taylor and Ivry proposed that Smith's two state model reflected the explicit and implicit components (Taylor and Ivry 2011), and McDougle, Bond et al. (2015) provided experimental support for this proposal. A second advantage of the linearity assumption is that allows researchers to measure only one component and use it to calculate the unmeasured component. Thus, 
researchers can measure the explicit component using the report and then calculate implicit as the difference between the report and the actual movement (Taylor, Krakauer et al. 2014). However, the fact that different measures of explicit and implicit often yield different results (as discussed in the previous paragraph) represents a challenge to the assumption of linearity. It is further challenged by many studies showing that implicit and explicit do not add up to total behavior (Hegele and Heuer 2010, Hegele and Heuer 2013, Heuer and Hegele 2015, Werner, van Aken et al. 2015, Schween and Hegele 2017, Schween, Taylor et al. 2018, Modchalingam, Vachon et al. 2019, Werner, Strüder et al. 2019). Studies using report tend to show more linearity than those that do not.

There are two possible explanations for lack of consistency across measures and failure of linearity. Our measures could be biased providing us with wrong estimates of the underlying processes. Explicit and/or implicit may also comprise multiple subcomponents. These subcomponents may have

Table 3 Overview of articles using the seven major methodologies for quantifying explicit and/or implicit processes. Shown are the differences and agreements of these articles on different features of the processes: The first three columns are taken from Table 2; the last four columns show key characteristics of the two processes.

\begin{tabular}{|c|c|c|c|c|c|c|c|}
\hline Article & $\begin{array}{l}\text { Explicit can be } \\
\text { controlled }\end{array}$ & $\begin{array}{l}\text { Explicit can be } \\
\text { reported }\end{array}$ & $\begin{array}{l}\text { Explicit } \\
\text { requires } \\
\text { resources }\end{array}$ & $\begin{array}{l}\text { Implicit is } \\
\text { slow \& } \\
\text { explicit is fast } \\
\text { (timescale) }\end{array}$ & $\begin{array}{l}\text { Implicit is task } \\
\text { independent }\end{array}$ & $\begin{array}{l}\text { Different } \\
\text { measures of } \\
\text { processes } \\
\text { agree }\end{array}$ & $\begin{array}{c}\text { Explicit \& } \\
\text { implicit } \\
\text { linearly related }\end{array}$ \\
\hline $\begin{array}{l}\text { Mazzoni \& Krakauer } \\
2006\end{array}$ & $\checkmark$ & & & $\checkmark$ & & & $\checkmark$ \\
\hline $\begin{array}{l}\text { Werner et al. (2015 \& } \\
\text { 2019) }\end{array}$ & $\checkmark$ & & & & & & $x$ \\
\hline $\begin{array}{l}\text { Hever \& Hegele }(2008, \\
2014,2015) \text { Hegele \& } \\
\text { Hever }(2010,2013)\end{array}$ & $\checkmark$ & $\checkmark$ & & & $\checkmark$ & $x$ & $x$ \\
\hline $\begin{array}{l}\text { Sülzenbrück \& Hever } \\
2009\end{array}$ & & $\checkmark$ & & & & & $\checkmark$ \\
\hline $\begin{array}{l}\text { Schween et al. }(2017, \\
2018)\end{array}$ & & $\checkmark$ & & & $\checkmark$ & & $\mathrm{x}$ \\
\hline $\begin{array}{l}\text { Taylor et al. }(2010,2011 \text {, } \\
2014)\end{array}$ & $\checkmark$ & $\checkmark$ & & $\checkmark$ & $\checkmark$ & $x$ & $\checkmark$ \\
\hline Bond ef al. 2015 & & & & $\checkmark$ & $\checkmark$ & $x$ & assumed \\
\hline Brudner et al. 2016 & & $\checkmark$ & & $\checkmark$ & & $\checkmark$ & assumed \\
\hline De Brouwer et al. 2018 & $\checkmark$ & $\checkmark$ & & $\checkmark$ & & $x$ & assumed \\
\hline Benson ef al. 2011 & & & $\checkmark$ & $\checkmark$ & & & $\checkmark$ \\
\hline Haith et al. 2015 & & & $\checkmark$ & $\checkmark$ & & & $\checkmark$ \\
\hline $\begin{array}{l}\text { Kim et al. 2018, Kim et al. } \\
2019\end{array}$ & $\checkmark$ & & & $\checkmark$ for implicit & $\mathrm{x}$ & $x$ & \\
\hline $\begin{array}{l}\text { Morehead et al. (2015 \& } \\
\text { 2017) }\end{array}$ & $\checkmark$ & $\checkmark$ & & $\checkmark$ & $\checkmark$ & $\checkmark$ & assumed \\
\hline $\begin{array}{l}\text { McDougle et al. (2015 \& } \\
2019)\end{array}$ & & $\checkmark$ & $\checkmark$ & $\checkmark$ & $x$ & & assumed \\
\hline $\begin{array}{l}\text { Modchalingam et al. } \\
(2018)\end{array}$ & $\checkmark$ & & & & $\checkmark$ & & $x$ \\
\hline Leow et al. (2018 \& 2019) & $\checkmark$ & $\checkmark$ & $\checkmark$ & $\checkmark$ & $x$ & $x$ & assumed \\
\hline Yin et al. (2020) & $\checkmark$ & $\checkmark$ & & $\checkmark$ & $x$ & $\mathrm{x}$ & assumed \\
\hline Maresch \& Donchin 2020 & $\checkmark$ & $\checkmark$ & & $\checkmark$ & & $x$ & assumed \\
\hline Wilterson \& Taylor (2019) & $\checkmark$ & $\checkmark$ & & $\checkmark$ & & $x$ & assumed \\
\hline Myiamoto et al. (2020) & & $\checkmark$ & & & $x$ & & assumed \\
\hline
\end{tabular}


different expression in the different measures. Either possibility would lead to inconsistent estimates that depend on condition and would consequently produce measures that do not add up to behavior.

\section{Explanations from outside the motor field box}

In order to bring some clarity to the failure of linearity of the explicit and implicit systems in the motor systems, it can be helpful to explore the issue more broadly. Explicit and implicit processes and knowledge have a much longer history in the cognitive literature than they do in the motor field. We explore here the way that the cognitive literature has addressed issues of measurement of and interaction between the different processes. We will then use the insight gained to suggest a path forward for the study of explicit and implicit motor adaptation.

As mentioned in the previous section, apparent non-linearity can arise due to a true underlying failure of linearity or due to bias in measurements. To explore the first possibility, we take as an example Jacoby's influential model of process dissociation, which assumes independent processes that combine linearly to produce behavior (Jacoby 1991). The process dissociation model aims at estimating the independent contributions of explicit and implicit memory systems by comparing performance in two conditions during the same task. Jacoby originally developed the procedure using a word stem completion task, where subjects were either asked to complete the stem using previously presented words (inclusion), or to avoid using these words (exclusion). The underlying rationale was that if subjects indeed use a previously presented word to fill the stem in the inclusion condition, this could either reflect an explicit process (where subjects knowingly use the retrieved word), an implicit process (where subjects don't explicitly remember the word, but are biased towards using it due to implicit retrieval), or chance (i.e., baseline probability to provide the word). In the exclusion condition, on the other hand, if subjects provide the previously presented word, it cannot represent explicit processes (as they were directly instructed not to use that word). A previously presented word could accordingly either come from implicit memory or chance (similar to the rationale of the process dissociation procedure used in adaptation tasks). The process dissociation procedure has been adapted to many other fields and paradigms, such as sequence learning (Destrebecqz and Cleeremans 2001, Karabanov and Ullén 2008), implicit social cognition tasks (Payne 2008, Payne and Bishara 2009, Stewart, von Hippel et al. 2009), decision making tasks (Liu, Johnson et al. 2005, Damian and Sherman 2013) and finally also in adaptation. 
However, the assumption of linearity underlying the PDP faces two major challenges. First, it assumes that the same amount of implicit knowledge is expressed in both the inclusion and exclusion conditions. Without the assumption of invariance, there is no way to compare across task conditions and no way to assess the amount of explicit and implicit knowledge. The second major assumption underlying the PDP is that the amount of explicit and implicit knowledge expressed within a specific trial is not correlated. If fluctuation in access to knowledge is correlated - more explicit knowledge on a specific trial tends to come with more (or less) implicit knowledge on that trial - estimates of explicit and implicit will be inaccurate (Curran and Hintzman 1995). It turns out that both assumptions are violated in practice (Dodson and Johnson 1996, Barth, Stahl et al. 2019), a fact that has been extensively discussed (Curran and Hintzman 1995, Curran and Hintzman 1997, Jacoby, Begg et al. 1997). From this example we learn that there are multiple ways that linearity of the underlying processes can be violated and that we must be very careful in how we estimate the two processes.

The alternative is that underlying explicit and implicit processes do combine additively to produce behavior (what we call linearity), but that linearity is apparently violated because our measures of the explicit or implicit process are biased. In some situations, our real knowledge overflows our ability to report that knowledge, leading to underestimation of explicit knowledge and apparently sublinear combination of explicit and implicit knowledge; in other situations, confabulated feelings of control and understanding may cause overestimation of explicit knowledge and superlinear combination of the explicit and implicit.

Claims of overflow rely on situations where we consciously experience more than we can report (Block, 2011). This seems to be the case in a classic experimental paradigm developed in 1960 by George Sperling (Sperling 1960). In this paradigm, subjects are presented with an array of items (e.g., letters), organized in rows, and are asked to report as many items as possible after the array is no longer shown. Subjects are typically able to only remember 3-4 items (out of 12 in the classic Sterling task, where 3 rows of 4 letters were presented). Critically however, when a post-cue is presented, signaling which row should be reported, subjects are able to remember most items in that row. This seems to suggest that they store a representation of most letters, although being able to report only a few of them, in line with the overflow argument (Block 2011). Similar findings have been obtained with change detection, where subjects were able to detect changes in a display using a cue that appeared a second or more later (Landman, Spekreijse et al. 2003, Sligte, Scholte et al. 2008). A different sort of overflow can be seen in experiments where subjects report color diversity in the uncued rows of a 
Sperling display, although unable to report the identity of the letters (Bronfman, Brezis et al. 2014). Notably however, neither the idea of overflow nor the claim that these examples demonstrate overflow are universally accepted. The argument is still ongoing, both on a theoretical level (Kouider, De Gardelle et al. 2010, Block 2014, Cohen, Dennett et al. 2016, Phillips 2016, Haun, Tononi et al. 2017) and an experimental one (Vandenbroucke, Sligte et al. 2014, Ward, Bear et al. 2016, Otten, Pinto et al. 2017, Cohen, Botch et al. 2020, Cohen and Rubenstein 2020). What we can learn from this literature, though, is that we should not overlook the possibility that our estimates of explicit knowledge are biased by a gap between one's experience, or knowledge, and the ability to report it. In the motor field, this possible discrepancy has recently been highlighted by Hadjiosif and Krakauer (2020).

On the other side of the continuum are claims that report might actually be overestimating perception rather than underestimating it. Indeed, there is some indication that verbal report can be confabulated such that it does not reflect explicit knowledge at all. Subjects can report percepts for non-existent stimuli. Recent examples include subjects who report seeing false shapes or texture (Otten, Pinto et al. 2017), or fail to notice surprisingly big color changes (Cohen, Botch et al. 2020, Cohen and Rubenstein 2020). This gap between the visual stimuli and report can have two explanations. Perception "may fill" in what is missing in the stimuli to create an actual experience distinct from the stimuli. Alternatively, the experience may be appropriate to the visual stimuli but become "inflated" in the report. These possibilities can be difficult to distinguish; however, Odegaard, Chang et al. (2018), using carefully controlled experiments, show that two separate and parallel phenomena - metacognitive deficits and decisional biases - can drive inflation of reports. Indeed, confabulation can reach surprising extremes, such as those in the literature on memory (for review, see Metcalf, Langdon et al. (2007)). To give just one example, Kassin and Kiechel (1996) falsely accused subjects of damaging a computer by pressing the wrong button. Despite the fact that none of them did press the wrong button, subjects readily admitted the "crime", internalizing guilt for it and even confabulating details consistent with that belief (this work was more recently replicated by Horselenberg, Merckelbach et al. (2003)). Thus, reports may overestimate or underestimate conscious experience; either way, they may be biased.

\section{How do the cognitive ideas affect our views on adaptation?}

The cognitive literature thus poses at least two challenges to common assumptions in the field of motor learning: the assumption that explicit and implicit processes additively explain behavior and the 
assumption that explicit processes are accurately measured using report. Together, these represent a significant challenge to the methodology used to study explicit and implicit motor adaptation.

A related challenge has recently been raised by Hadjiosif and Krakauer (2020) who suggest that the experimental methods may be imposing the explicit/implicit distinction by assuming the existence of the underlying processes they purport to measure. In fact, they suggest, there may not be distinguishable underlying explicit and implicit biological processes. They argue for an alternative approach where different adaptation processes are distinguished by their sensitivity to the reaction time rather than the extent to which subjects are or are not explicitly aware of them. While we recognize the concern, the literature reviewed above reveals a number of robust results that allow characterizations of distinct explicit and implicit processes of adaptation valid across multiple methods of measurement. Consistent behavioral results do not necessarily reflect real, distinct underlying biological processes, but they do serve as a basis for exploring this possibility. Indeed, a growing literature addresses specific neural signifiers of explicit and implicit adaptation (Savoie, Thénault et al. 2018, Jahani, Schwey et al. 2020, Jonker, van der Vliet et al. 2020).

Indeed, there is ample evidence that our explicit motor intentions do affect our movements. For example, explicit knowledge is known to improve learning. This can happen when subjects are informed about the rotation (or perturbation of any kind) prior to learning (Mazzoni and Krakauer 2006, Taylor and Ivry 2011, Werner, van Aken et al. 2015, Modchalingam, Vachon et al. 2019). It's also clear from exclusion trials where a specific change in strategy changes behavior on the following trial (Bromberg, Donchin et al. 2019, Maresch, Werner et al. 2020). Also supporting this is converging evidence that increasing explicit knowledge of the task leads to increased learning rate (Bromberg, Donchin et al. 2019, Langsdorf, Maresch et al. 2019, Maresch, Werner et al. 2020). Thus, while our measures of explicit adaptation may be biased in overestimating the contribution of explicit processes to adaptation, is seems clear that there should be some degree of explicit processes playing a role in adaptation behavior. On this basis, we argue that our explicit intention in our motor behavior cannot be completely confabulated.

Bias is especially troublesome in the absence of any strategy for knowing what would serve to calibrate our measures. Without a "true" measure of implicit and explicit processes, we cannot know the scope of this bias. The cognitive literature deals much more extensively with bias in report-based measures than bias in control-based measures such as exclusion. Thus, while we know that bias is a problem for report-based measures, there is less evidence available regarding control-based measures. To make 
progress, we must develop an extensive database of adaptation using multiple measures taken simultaneously in a broad range of conditions (our recent paper represents an effort in this direction, Maresch et al., 2020). We must also develop alternative, realistic and parsimonious models of how different components of explicit and implicit adaptation interact. Sufficient data and a rich set of alternative models will allow us to identify a model that can explain how and why measures differ in different conditions and relate this to the phenomena we see.

Our models will need to include multiple forms of explicit and implicit adaptation that develop in different conditions. Some of these models will posit the presence of multiple explicit components: McDougle and Taylor (2019) suggested that explicit adaptation includes a cached component and a mental rotation component; our 2020 paper proposes a distinction between the ability to control behavior through explicit intention and the ability to explicitly access that intention for report (Maresch, Werner et al. 2020). Similarly, implicit adaptation could also be made up of different components. Kim, Parvin et al. (2019) suggested that implicit adaptation could be divided into a labile and a stable component, and Miyamoto, Wang et al. (2020) proposed a distinction between sensoryprediction driven and performance-error driven frequencies in implicit adaptation. The authors further suggested a compensatory role of implicit adaptation, in which it compensates for errors in explicit strategy. Another possibility is the combination of model-based and model-free mechanisms that together combine to form motor learning (Haith and Krakauer 2013). Different compositions of explicit and implicit adaptation would necessarily have consequences for the interaction between the two processes, and for their expression in the different measures, as proposed by Maresch, Werner et al. (2020)(Fig. 10). Do these different models reflect different perspectives on the same underlying set of components? Alternatively, is explicit adaptation a complex mixture of a multiplicity of underlying processes that cannot be cleanly delineated? Our field needs an answer to this question.

Of course, the model must explain both the interaction of the multiple components and how they combine to produce behavior. Non-linearities in the behavior need to be characterized and the model needs to address them. This will require measuring implicit and explicit in the same experiment. Ultimately, our gold standard will be a set of experiments consistent with our model that highlight the effects of each component of adaptation in a coherent fashion. 


\section{Conclusions}

Our survey of the literature of explicit and implicit motor adaptation reveals some surprisingly robust findings that characterize the explicit and implicit adaptation systems. Despite this convergence, methodological issues need to be addressed. Our review focused on two of them: the use of multiple incompatible measures of explicit and implicit adaptation, and the assumption that explicit and implicit adaptation combine linearly. We sought to interpret these concerns in the context of the larger literature in cognitive science on explicit and implicit knowledge, which showed that similar concerns arise in the broader context and that they cannot be ignored. The cognitive literature questions the reliability of measures of both explicit and implicit knowledge, raising concerns about possible biases. Further, while the explicit and implicit systems may combine linearly, this is difficult to test without making restrictive assumptions that may not hold. Applying these insights in the motor field, we propose that bias, particularly of the report measure, must be addressed and may also lead to apparent non-linearities. This may well go hand-in-hand with the idea of multiple components or levels of explicit knowledge as suggested both in the motor field (McDougle and Taylor 2019, Maresch, Werner

et al. 2020) and in the cognitive fields (Block 1995) anyone else?). In conclusion, additional experimental data as well as more sophisticated models are needed for the field to make progress towards a coherent account of the contributions of explicit and implicit processes to adaptation. There is much still to be done.

\section{Acknowledgements}

We would like to thank Dr. Matthias Hegele and Lisa-Marie Langsdorf for helpful discussions.

This work is part of the PACE project (itn-pace.eu), which received funding from the European Union's Horizon 2020 research and innovation program under the Marie Skłodowska-Curie grant agreement No 642961.

No conflicts of interest, financial or otherwise, are declared by the authors.

\section{References}


Anguera, J. A., P. A. Reuter-Lorenz, D. T. Willingham and R. D. Seidler (2010). "Contributions of spatial working memory to visuomotor learning." Journal of cognitive neuroscience 22(9): 1917-1930. Barth, M., C. Stahl and H. Haider (2019). "Assumptions of the process-dissociation procedure are violated in implicit sequence learning." Journal of Experimental Psychology: Learning, Memory, and Cognition 45(4): 641.

Bernstein, N. (1967). "The co-ordination and regulation of movements: Conclusions towards the Study of Motor Co-ordination." Biodynamics of Locomotion: 104-113.

Block, N. (1995). "On a confusion about a function of consciousness." Behavioral and brain sciences 18(2): 227-247.

Block, N. (2011). "Perceptual consciousness overflows cognitive access." Trends in cognitive sciences 15(12): 567-575.

Block, N. (2014). "Rich conscious perception outside focal attention." Trends in Cognitive Sciences 18(9): 445-447.

Bond, K. M. and J. A. Taylor (2015). "Flexible explicit but rigid implicit learning in a visuomotor adaptation task." L Neurophysiol 113(10): 3836-3849.

Bond, K. M. and J. A. Taylor (2017). "Structural Learning in a Visuomotor Adaptation Task Is Explicitly Accessible." eNeuro 4(4).

Bromberg, Z., O. Donchin and S. Haar (2019). "Eye movements during visuomotor adaptation represent only part of the explicit learning." bioRxiv: 724864.

Bronfman, Z. Z., N. Brezis, H. Jacobson and M. Usher (2014). "We See More Than We Can Report: "Cost Free" Color Phenomenality Outside Focal Attention." Psychological science 25(7): 1394-1403.

Brudner, S. N., N. Kethidi, D. Graeupner, R. B. Ivry and J. A. Taylor (2016). "Delayed feedback during sensorimotor learning selectively disrupts adaptation but not strategy use." Journal of neurophysiology 115(3): 1499-1511.

Chun, M. M. and Y. Jiang (1998). "Contextual cueing: Implicit learning and memory of visual context guides spatial attention." Cognitive psychology 36(1): 28-71.

Cleeremans, A., A. Destrebecqz and M. Boyer (1998). "Implicit learning: News from the front." Trends in cognitive sciences 2(10): 406-416.

Cohen, M. A., T. L. Botch and C. E. Robertson (2020). "The limits of color awareness during active, real-world vision." Proceedings of the National Academy of Sciences.

Cohen, M. A., D. C. Dennett and N. Kanwisher (2016). "What is the bandwidth of perceptual experience?" Trends in cognitive sciences 20(5): 324-335. 
Cohen, M. A. and J. Rubenstein (2020). "How much color do we see in the blink of an eye?" Cognition 200: 104268.

Curran, T. and D. L. Hintzman (1995). "Violations of the independence assumption in process dissociation." Journal of Experimental Psychology: Learning, Memory, and Cognition 21(3): 531.

Curran, T. and D. L. Hintzman (1997). "Consequences and causes of correlations in process dissociation."

Damian, R. I. and J. W. Sherman (2013). "A process-dissociation examination of the cognitive processes underlying unconscious thought." Journal of Experimental Social Psychology 49(2): 228237.

de Brouwer, A. J., J. P. Gallivan and J. R. Flanagan (2018). "Visuomotor feedback gains are modulated by gaze position." Journal of neurophysiology 120(5): 2522-2531.

Dehaene, S., J.-P. Changeux, L. Naccache, J. Sackur and C. Sergent (2006). "Conscious, preconscious, and subliminal processing: a testable taxonomy." Trends in cognitive sciences 10(5): 204-211.

DeKeyser, R. (2008). "11 Implicit and Explicit Learning." The handbook of second language acquisition 27: 313.

Destrebecqz, A. and A. Cleeremans (2001). "Can sequence learning be implicit? New evidence with the process dissociation procedure." Psychonomic bulletin \& review 8(2): 343-350.

Dharani, K. (2014). The biology of thought: A neuronal mechanism in the generation of thought-A new molecular model, Academic Press.

Dodson, C. S. and M. K. Johnson (1996). "Some problems with the process-dissociation approach to memory." Journal of Experimental Psychology: General 125(2): 181.

Eversheim, U. and O. Bock (2001). "Evidence for processing stages in skill acquisition: a dual-task study." Learning \& Memory 8(4): 183-189.

Fernandez-Ruiz, J., W. Wong, I. T. Armstrong and J. R. Flanagan (2011). "Relation between reaction time and reach errors during visuomotor adaptation." Behavioural brain research 219(1): 8-14.

Fitts, P. M. and M. I. Posner (1967). "Human performance."

Georgopoulos, A. and J. Massey (1987). "Cognitive spatial-motor processes." Experimental Brain Research 65(2): 361-370.

Graf, P. and D. L. Schacter (1985). "Implicit and explicit memory for new associations in normal and amnesic subjects." Journal of Experimental Psychology: Learning, memory, and cognition 11(3): 501. Hadjiosif, A. M. and J. W. Krakauer (2020). "The explicit/implicit distinction in studies of visuomotor learning: conceptual and methodological pitfalls." European Journal of Neuroscience. 
Haith, A. M., D. M. Huberdeau and J. W. Krakauer (2015). "The influence of movement preparation time on the expression of visuomotor learning and savings." L Neurosci 35(13): 5109-5117.

Haith, A. M. and J. W. Krakauer (2013). Model-based and model-free mechanisms of human motor learning. Progress in motor control, Springer: 1-21.

Hassin, R. R. (2013). "Yes it can: On the functional abilities of the human unconscious." Perspectives on Psychological Science 8(2): 195-207.

Haun, A. M., G. Tononi, C. Koch and N. Tsuchiya (2017). "Are we underestimating the richness of visual experience?" Neuroscience of Consciousness 2017(1): niw023.

Hegele, M. and H. Heuer (2010). "Implicit and explicit components of dual adaptation to visuomotor rotations." Conscious Cogn 19(4): 906-917.

Hegele, M. and H. Heuer (2013). "Age-related variations of visuomotor adaptation result from both the acquisition and the application of explicit knowledge." Psychology and aging 28(2): 333.

Heuer, H. and M. Hegele (2015). "Explicit and implicit components of visuo-motor adaptation: An analysis of individual differences." Consciousness and cognition 33: 156-169.

Horselenberg, R., H. Merckelbach and S. Josephs (2003). "Individual differences and false confessions: A conceptual replication of Kassin and Kiechel (1996)." Psychology, Crime and law 9(1): 1-8.

Huberdeau, D. M., J. W. Krakauer and A. M. Haith (2019). "Practice induces a qualitative change in the memory representation for visuomotor learning." Journal of neurophysiology 122(3): 1050-1059. Jacoby, L. L. (1991). "A process dissociation framework: Separating automatic from intentional uses of memory." Journal of memory and language 30(5): 513-541.

Jacoby, L. L., I. M. Begg and J. P. Toth (1997). "In defense of functional independence: Violations of assumptions underlying the process-dissociation procedure?".

Jahani, A., A. Schwey, P.-M. Bernier and N. Malfait (2020). "Spatially distinct beta-band activities reflect implicit sensorimotor adaptation and explicit re-aiming strategy." Journal of Neuroscience 40(12): 2498-2509.

Jonker, Z. D., R. van der Vliet, G. Maquelin, J. van der Cruijsen, G. M. Ribbers, R. W. Selles, O. Donchin and M. A. Frens (2020). "Individual differences in frontal midline theta activity during visuomotor adaptation are related to execution noise." $\underline{\text { bioRxiv. }}$

Karabanov, A. and F. Ullén (2008). "Implicit and explicit learning of temporal sequences studied with the process dissociation procedure." Journal of Neurophysiology 100(2): 733-739.

Kassin, S. M. and K. L. Kiechel (1996). "The social psychology of false confessions: Compliance, internalization, and confabulation." Psychological science 7(3): 125-128. 
Kim, H. E., D. E. Parvin and R. B. Ivry (2019). "The influence of task outcome on implicit motor learning." eLife 8: e39882.

Kouider, S., V. De Gardelle, J. Sackur and E. Dupoux (2010). "How rich is consciousness? The partial awareness hypothesis." Trends in cognitive sciences 14(7): 301-307.

Kouider, S. and S. Dehaene (2007). "Levels of processing during non-conscious perception: a critical review of visual masking." Philosophical Transactions of the Royal Society B: Biological Sciences 362(1481): 857-875.

Koziol, L. F. and D. E. Budding (2012). Procedural Learning. Encyclopedia of the Sciences of Learning. N. M. Seel. Boston, MA, Springer US: 2694-2696.

Krakauer, J. W. and P. Mazzoni (2011). "Human sensorimotor learning: adaptation, skill, and beyond." Current opinion in neurobiology 21(4): 636-644.

Krakauer, J. W., Z. M. Pine, M. F. Ghilardi and C. Ghez (2000). "Learning of visuomotor transformations for vectorial planning of reaching trajectories." L Neurosci 20(23): 8916-8924.

Lamme, V. A. (2006). "Towards a true neural stance on consciousness." Trends in cognitive sciences 10(11): 494-501.

Landman, R., H. Spekreijse and V. A. Lamme (2003). "Large capacity storage of integrated objects before change blindness." Vision research 43(2): 149-164.

Langsdorf, L., J. Maresch, M. Hegele, S. D. McDougle and R. Schween (2019). "Prolonged reaction times eliminate residual errors in visuomotor adaptation." bioRxiv.

Leow, L. A., R. Gunn, W. Marinovic and T. J. Carroll (2017). "Estimating the implicit component of visuomotor rotation learning by constraining movement preparation time." L Neurophysiol 118(2): 666-676.

Liu, S. S., K. F. Johnson and K. F. Johnson (2005). "The automatic country-of-origin effects on brand judgments." Journal of advertising 34(1): 87-97.

Maresch, J., S. Werner and O. Donchin (2020). "Methods matter: your measures of explicit and implicit processes in visuomotor adaptation affect your results." bioRxiv: 702290.

Mazzoni, P. and J. W. Krakauer (2006). "An implicit plan overrides an explicit strategy during visuomotor adaptation." L Neurosci 26(14): 3642-3645.

McDougle, S. D., K. M. Bond and J. A. Taylor (2015). "Explicit and Implicit Processes Constitute the Fast and Slow Processes of Sensorimotor Learning." J Neurosci 35(26): 9568-9579.

McDougle, S. D. and J. A. Taylor (2019). "Dissociable cognitive strategies for sensorimotor learning." Nat Commun 10(1): 40. 
Metcalf, K., R. Langdon and M. Coltheart (2007). "Models of confabulation: A critical review and a new framework." Cognitive Neuropsychology 24(1): 23-47.

Miyamoto, Y. R., S. Wang and M. A. Smith (2020). "Implicit adaptation compensates for erratic explicit strategy in human motor learning." Nature Neuroscience 23(3): 443-455.

Modchalingam, S., C. M. Vachon, B. M. 't Hart and D. Y. Henriques (2019). "The effects of awareness of the perturbation during motor adaptation on hand localization." PloS one 14(8): e0220884.

Modchalingam, S., C. M. Vachon, B. Marius't Hart and D. Y. Henriques (2019). "The effects of awareness of the perturbation during motor adaptation on hand localization." PloS one 14(8).

Morehead, J. R., J. A. Taylor, D. E. Parvin and R. B. Ivry (2017). "Characteristics of Implicit Sensorimotor Adaptation Revealed by Task-irrelevant Clamped Feedback." L Cogn Neurosci 29(6): 1061-1074.

Mudrik, L., N. Faivre and C. Koch (2014). "Information integration without awareness." Trends in cognitive sciences 18(9): 488-496.

Newell, B. R. and D. R. Shanks (2014). "Unconscious influences on decision making: A critical review." Behavioral and Brain Sciences 37(1): 1-19.

Nosek, B. A. (2007). "Implicit-explicit relations." Current Directions in Psychological Science 16(2): 65-69.

Odegaard, B., M. Y. Chang, H. Lau and S.-H. Cheung (2018). "Inflation versus filling-in: why we feel we see more than we actually do in peripheral vision." Philosophical Transactions of the Royal Society B: Biological Sciences 373(1755): 20170345.

Otten, M., Y. Pinto, C. L. Paffen, A. K. Seth and R. Kanai (2017). "The uniformity illusion: Central stimuli can determine peripheral perception." Psychological Science 28(1): 56-68.

Payne, B. K. (2008). "What mistakes disclose: A process dissociation approach to automatic and controlled processes in social psychology." Social and Personality Psychology Compass 2(2): 10731092.

Payne, B. K. and A. J. Bishara (2009). "An integrative review of process dissociation and related models in social cognition." European review of social psychology 20(1): 272-314.

Perugini, M. (2005). "Predictive models of implicit and explicit attitudes." British Journal of Social Psychology 44(1): 29-45.

Phillips, I. (2016). "No watershed for overflow: Recent work on the richness of consciousness." Philosophical Psychology 29(2): 236-249. 
Reber, A. S. (1989). "Implicit learning and tacit knowledge." Journal of experimental psychology: General 118(3): 219.

Rothkirch, M. and G. Hesselmann (2017). "What we talk about when we talk about unconscious processing-A plea for best practices." Frontiers in Psychology 8: 835.

Savoie, F.-A., F. Thénault, K. Whittingstall and P.-M. Bernier (2018). "Visuomotor prediction errors modulate EEG activity over parietal cortex." Scientific reports 8(1): 12513.

Schween, R. and M. Hegele (2017). "Feedback delay attenuates implicit but facilitates explicit adjustments to a visuomotor rotation." Neurobiol Learn Mem 140: 124-133.

Schween, R., J. A. Taylor and M. Hegele (2018). "Plan-based generalization shapes local implicit adaptation to opposing visuomotor transformations." Journal of neurophysiology 120(6): 2775-2787. Shadmehr, R. and F. A. Mussa-Ivaldi (1994). "Adaptive representation of dynamics during learning of a motor task." L Neurosci 14(5 Pt 2): 3208-3224.

Sligte, I. G., H. S. Scholte and V. A. Lamme (2008). "Are there multiple visual short-term memory stores?" PLOS one 3(2): e1699.

Smith, M. A., A. Ghazizadeh and R. Shadmehr (2006). "Interacting adaptive processes with different timescales underlie short-term motor learning." PLoS Biol 4(6): e179.

Sperling, G. (1960). "The information available in brief visual presentations." Psychological monographs: General and applied 74(11): 1.

Stewart, B. D., W. von Hippel and G. A. Radvansky (2009). "Age, race, and implicit prejudice: Using process dissociation to separate the underlying components." Psychological Science 20(2): 164-168.

Sülzenbrück, S. and H. Heuer (2009). "Functional independence of explicit and implicit motor adjustments." Consciousness and Cognition 18(1): 145-159.

Taylor, J. A. and R. B. Ivry (2011). "Flexible cognitive strategies during motor learning." PLoS Comput Biol 7(3): e1001096.

Taylor, J. A., J. W. Krakauer and R. B. Ivry (2014). "Explicit and implicit contributions to learning in a sensorimotor adaptation task." L Neurosci 34(8): 3023-3032.

Timmermans, B. and A. Cleeremans (2015). "How can we measure awareness? An overview of current methods." Behavioural methods in consciousness research: 21-46.

van Gaal, S., F. P. De Lange and M. X. Cohen (2012). "The role of consciousness in cognitive control and decision making." Frontiers in human neuroscience 6: 121. 
Vandenbroucke, A. R., I. G. Sligte, A. B. Barrett, A. K. Seth, J. J. Fahrenfort and V. A. Lamme (2014). "Accurate metacognition for visual sensory memory representations." Psychological science 25(4): 861-873.

Ward, E. J., A. Bear and B. J. Scholl (2016). "Can you perceive ensembles without perceiving individuals?: The role of statistical perception in determining whether awareness overflows access." Cognition 152: 78-86.

Welch, R. B. (1986). "Adaptation of space perception." Handbook of perception and human performance $\mathbf{1}(24)$.

Werner, S., H. K. Strüder and O. Donchin (2019). "Intermanual transfer of visuomotor adaptation is related to awareness." PloS one 14(9): e0220748.

Werner, S., B. C. van Aken, T. Hulst, M. A. Frens, J. N. van der Geest, H. K. Struder and O. Donchin (2015). "Awareness of sensorimotor adaptation to visual rotations of different size." PLoS One 10(4): e0123321.

Wilterson, S. A. and J. A. Taylor (2019). "Implicit visuomotor adaptation remains limited after several days of training." BioRxiv: 711598.

Yin, C. and K. Wei (2020). "Savings in sensorimotor adaptation without an explicit strategy." Journal of Neurophysiology 123(3): 1180-1192. 